\title{
A DEM Generalized Kelvin Contact model for Predicting Creep of Asphalt Mixtures
}

\author{
Gustavo Câmara *, Nuno Azevedo ${ }^{1 \dagger}$ and Rui Micaelo ${ }^{2 \dagger}$ \\ * DEC, FCT, Universidade NOVA de Lisboa, Caparica, Portugal \\ e-mail: g.camara@campus.fct.unl.pt \\ ${ }^{1 \dagger}$ Portuguese National Laboratory for Civil Engineering \\ Avenida do Brasil, 101, Lisboa, Portugal \\ e-mail: nazevedo@lnec.pt \\ ${ }^{2 \dagger}$ DEC, FCT, Universidade NOVA de Lisboa, Caparica, Portugal \\ e-mail: ruilbm@fct.unl.pt
}

\begin{abstract}
Rigid particle models based on the discrete element method (DEM) have been adopted in the study of fracture and viscoelastic properties of mastic and asphalt mixtures [1]. In this work, a 3D particle model is proposed based on the Laguerre-Voronoi diagrams of the grain structure representing the asphalt mixture or of the mastic.
\end{abstract}

Usually, the DEM models of asphalt mixtures adopt a Burger's contact model to reproduce the known viscoelastic behaviour, following the methodology proposed in [2]. For a better agreement, between the numerical response obtained with a DEM model and the known experimental response of asphalt mixtures, a generalized Kelvin chain model (GK) has been developed within a PM framework.

Two different approaches are presented for a GK contact model. In the first approach a direct integration of the constitutive equations, using a centred difference scheme, similar to the adopted in [2] for the Burger's model equations is devised. In the second approach the proposed contact model is based on the incremental formulation of viscoelastic behaviour that has been proposed within the framework of the finite element method [3].

After a brief description of the GK based contact models, the proposed viscoelastic PM model is validated based on cyclic tests to determine the macroscopic mechanical behaviour of bituminous mastic available in the literature [4]. As so, a sinusoidal cyclic loading is applied for a frequency range varying from 10 to $0.1 \mathrm{~Hz}$. The tests are submitted to a strain control mechanism and the resulting stress is monitored. Compression creep tests are also modelled for different uniaxial compression values. After a previous calibration of the contact parameters, it is shown a good correlation between the numerical results obtained with the GK contact model and the experimental data when compared with the agreement obtained with the Burger's contact model. A comparison of the performance between the two proposed approaches is also presented.

Keywords: asphalt mixtures, viscoelastic, particle model, generalized Kelvin.

\section{REFERENCES}

[1] K. Chang and J. Meegoda, "Micromechanical simulation of hot mix asphalt", J Eng Mech-ASCE, 123(5), 495-503, (1997).

[2] A. C. Collop, A. Scarpas, C. Kasbergen and A. Bondt, "Development and finite element implementation of stress-dependent elastoviscoplastic constitutive model with damage for asphalt", Transportation Research Record, 96-104, (2003).

[3] Itasca, "Particle flow code in three dimensions, theory and background", Version 3.1, Itasca Consulting Group Inc., USA, (1999).

[4] H. Silva, "Characterization of the bituminous mastic and the aggregate-mastic bond", $\mathrm{PhD}$ thesis, Universidade do Minho (2005). 